Cytogenet Genome Res 1965;4:320

\title{
Author's Erratum
}

In the paper "A biometric analysis of the chromosomes of the marsupials - Macropus major, Macropus rufus and Potorous tridactylis" by Ruth Moore in the previous issue (Cytogenet Genome Res 4: 145-156, 1965) the abbreviation S. E. (standard error) should read standard deviation throughout. 